\title{
Quality Evaluation of Fiber-Optic Strain Data Acquired in Long-Term Bridge Monitoring
}

\author{
Mayuko Nishio* \\ Yokohama National University, 79-5 Tokiwadai, Hodogaya-ku, Yokohama 240-8501, Japan
}

(Received September 8, 2016; accepted November 7, 2016)

Keywords: structural health monitoring, fiber Bragg grating sensor, concrete bridge, long-term data, reliability, stability

The qualities of fiber Bragg grating (FBG) strain data acquired in the long-term structural health monitoring of an existing concrete bridge were evaluated in this study. The possible sources of errors in the acquired data were first considered on the basis of the principle of the FBG sensor and the details of the installation of system components: FBGs, cables, epoxy, FBG interrogator, and data acquisition system. The data reliability was first evaluated by a defined measure: the availability ratio of FBG peak wavelengths throughout the period of 2012-2014. It was then said that the high reliabilities of the FBG interrogator and data acquisition system were ensured; however, reliability reduction was observed in some FBGs. The stability was also evaluated on the basis of the accuracy and sensitivity. A characteristic disturbance in the data accuracy, which is the standard deviation of the acquired FBG peak wavelength, was recognized only in specific FBGs. Moreover, the attachment condition issues in some FBGs were also recognized by sensitivity analyses. The results and considerations in this paper indicated the importance of those data quality analyses for the appropriate use of the long-term data in structural condition assessment throughout the life of existing civil infrastructures.

\section{Introduction}

Sensor technologies are now expected to be used to enable the more reasonable operation of existing civil infrastructures. One strategy to take advantage of sensor technologies is to install structural health monitoring (SHM) systems. SHM is defined as the process of detecting structural changes due to damage or deterioration by comparing structural responses acquired by many kinds of sensors both temporally and spatially. The process requires not only sensor installation itself but also operational evaluation, data acquisition including sensor installation, comparative feature extraction, normalization, and statistical comparison or estimation for the diagnosis of structural conditions. It is thus important to understand the quality of acquired data such as reliability and stability on the basis of the error structure of the data to conduct those SHM processes successfully.

In addition, even though the sensor devices have been advanced, they could not be applied if the quality of the actual data, which are acquired under the installed condition in an actual bridge, is not appropriate including overspecification. Sugisaki et al. evaluated the sensor specifications for their specific monitoring strategy: dynamic data acquisition for the monitoring of railway bridge piers. ${ }^{(1)}$

*Corresponding author: e-mail: nishio@ynu.ac.jp http://dx.doi.org/10.18494/SAM.2017.1462 
They pointed out the importance of communication about required sensing performance between the sensor manufacturer and the user who conducts the SHM. Zhang and Aktan also presented the issues in the installation of a structural monitoring system. ${ }^{(2)}$ Here, the importance of designing and controlling both the specifications and the error was indicated to obtain the required performance as a monitoring system.

Meanwhile, the long-term SHM can provide much knowledge about the structural behavior during operation, and some algorithms have been proposed for comparing long-term data to the diagnosis of structural conditions; e.g., Refs. 3-6. The long-term SHM data in these studies showed the structural responses due to environmental and operational effects. They then indicated some algorithms to compare features extracted from the data for understanding structural conditions considering those effects. However, we have to also notice that there is the possibility of time variation in data quality, especially in the long-term data acquisition. There are only a few previous studies in which the quality of long-term bridge monitoring data was analyzed in detail. For instance, the study by Anderegg et al., in which the measurement uncertainty and reliability of 17 years worth of data acquired by fiber-optic bridge monitoring were discussed, yielded much knowledge about the required sensor specifications and the issues concerning system installation. ${ }^{(7)}$

The authors' group has also been working on a structural monitoring project of an existing bridge in Japan since 2011. The installed sensing system was constructed using fiber Bragg grating (FBG) sensors, and the acquired strain data have actually been used in the study to develop a structural condition assessment algorithm based on time-series analysis. ${ }^{(8)}$ However, the time variation in the data quality must be discussed for realizing a more advanced and applicable algorithm. In this paper, the author first summarizes the basic principle of the FBG sensor and explains the installation of the monitoring system onto the target bridge. After considerations about the possible error sources in the installed system, the data qualities are then analyzed and discussed from the viewpoints of long-term reliability and stability.

\section{2. $\quad$ Long-Term Bridge Monitoring Using Fiber-Optic Sensors}

\subsection{Principle of FBG sensor}

Fiber-optic sensors have been applied to SHMs of civil infrastructures. Its applications and procedures are generally summarized in Ref. 9. Although several kinds of sensing principles are existing in the fiber-optic sensors, the FBG sensors are widely adopted in actual bridge monitoring to acquire the data of strain, deformation, and dynamic properties.

The FBG is a periodic reflective index modulation fabricated by exposing ultraviolet light to the fiber core. It works as a reflection filter, as in the basic FBG principle illustrated in Fig. 1; when incident light with a broadband wavelength acts on a FBG, only the light with the Bragg wavelength $\lambda_{B}$ is reflected. The Bragg wavelength $\lambda_{B}$ is related to the grating period $A$ and the effective refractive index of the fiber, $n_{\text {eff }}$ as

$$
\lambda_{B}=2 n_{e f f} \Lambda
$$

The effective refractive index and the grating period then vary with the strain $\varepsilon$ and the temperature $\Delta T$ on the fiber. Both of them will change the Bragg wavelength through the expansion or contraction of the grating, through the photoelastic effect, and through the thermal dependence 


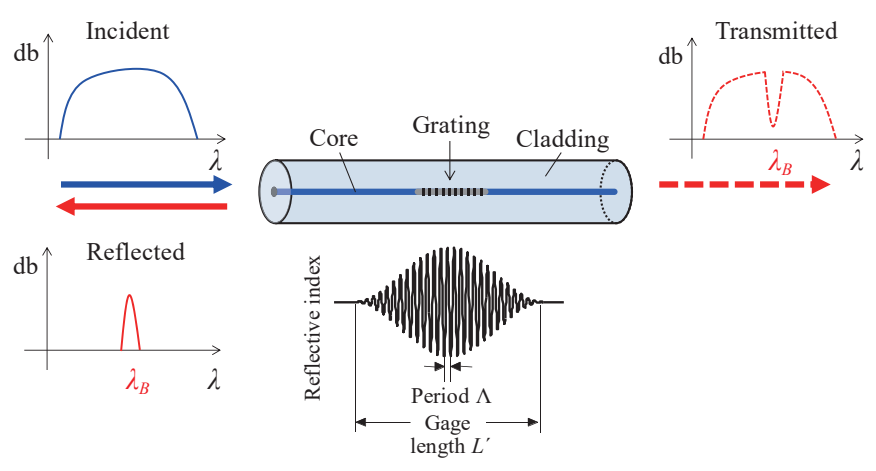

Fig. 1. (Color online) Principle of FBG sensor.

of the reflective index. At air temperature, the Bragg wavelength shift $\Delta \lambda_{B}$ can be described in the linear formula

$$
\frac{\Delta \lambda_{B}}{\lambda_{B}}=\left(1-p_{\varepsilon}\right) \varepsilon+\left(\alpha_{A}-\alpha_{n}\right) \Delta T
$$

where $p_{\varepsilon}$ is the strain-optic coefficient, $\alpha_{A}$ is the thermal expansion coefficient of the fiber, and $a_{n}$ is the fiber thermo-optic coefficient. These properties are known to be almost constant within different fibers. The coefficient for strain is $K_{\varepsilon}=0.0012 \mathrm{~nm} / \mu \varepsilon$, and that for temperature change is $K_{T}=$ $0.011 \mathrm{~nm} /{ }^{\circ} \mathrm{C}$ around a Bragg wavelength of $1550 \mathrm{~nm}$. Furthermore, the FBG sensors can realize multipoint measurement when more than one FBG with different Bragg wavelengths are fabricated on a single fiber.

\subsection{Target bridge and installed FBG monitoring system}

The bridge that we are working on in this study is a prestressed concrete (PC) box-girder bridge completed in 1973. The total length of the bridge is approximately $300 \mathrm{~m}$ with four continuous spans (65 $+85+85+65 \mathrm{~m}$ ), and its width is approximately $10 \mathrm{~m}$. One of the four spans is the target of monitoring in this study. As several kinds of sensors including FBG-based accelerometers and temperature sensors have actually been installed since 2011 for multivariate monitoring, the sensors on which the author focuses on in this paper are the FBG strain sensors, which have been used in an SHM study by the author's group. ${ }^{(8)}$

Eight strain sensing units, L1-1/2/3/4 and L2-1/2/3/4 indicated in Figs. 2(a) and 2(c), were attached on the inside surface of the box-girder web. Each sensor unit consisted of two FBGs, one was the "strain-FBG" that was completely attached to the structural surface, and the other was the "temp-FBG" that was placed just next to the "strain-FBG" for temperature correction, as shown in Fig. 2(b). The total number of FBGs was thus sixteen. Notice that the fibers were all single-mode fibers with a polyimide coating. Although the gage length of all FBGs was $L^{\prime}=5 \mathrm{~mm}$, each strainFBG was attached through a jig composed of two aluminum plate structures for realizing reliable epoxy attachments and for making the effective gage length $50 \mathrm{~mm}$, which was the appropriate gage length for concrete structures. All strain-FBGs were then installed in the longitudinal direction to capture the global deformation of the bridge. Notice that each unit was covered by an aluminum box for protection. 


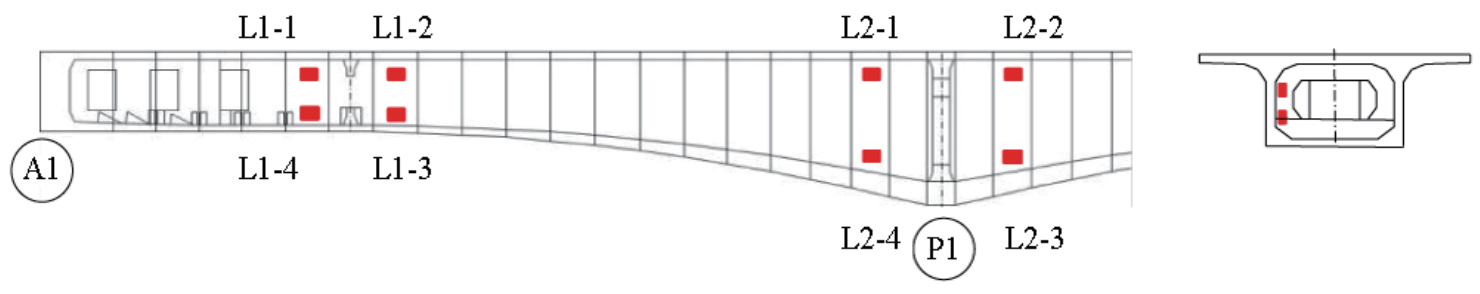

(a)

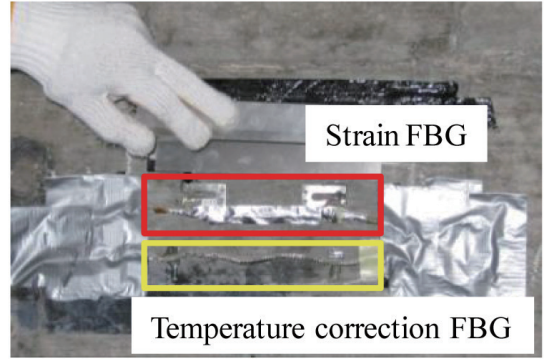

(b)

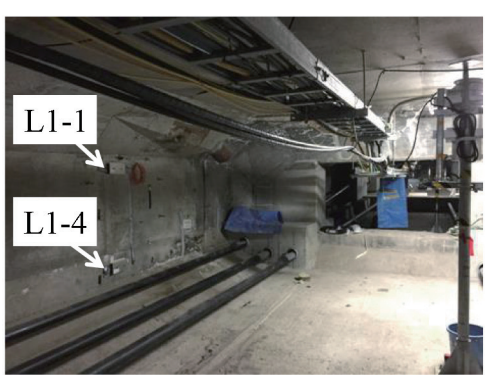

(c)

Fig. 2. (Color online) Installed fiber-optic monitoring system: (a) locations of FBG sensor units, (b) configuration of a sensor unit, and (c) interior of the box-girder of the target bridge.

$\Delta \lambda_{B}$ at the strain-FBG then includes the effects of both strain and temperature on the fiber, i.e., both the first and second terms of Eq. (2). Meanwhile, $\Delta \lambda_{B}$ at the temp-FBG is due to only the temperature effect if it is successfully installed. Therefore, only the strain effect on the fiber, the first term of Eq. (2), can be extracted by subtracting the Bragg wavelength shift at the temp-FBG from that at the strain-FBG at each sensing unit. The eight sensing units were connected to the FBG interrogator via two optical fiber cables, lines L1 and L2 as shown in Fig. 1(a), each line thus had four sensing units ( 8 FBGs) in series. The FBG interrogator has the specifications with the measurable wavelength range of $1520-1570 \mathrm{~nm}$ and with the wavelength reproducibility $<0.001$ $\mathrm{nm}$ in maximum, and the accuracy of $3 \sigma=0.001 \mathrm{~nm}(0.8 \mu \varepsilon$ in strain) is obtained when using an optical fiber with a length of $120 \mathrm{~km}$ in maximum. ${ }^{(10)}$ The data acquisition system was established using the LabVIEW software. In the long-term monitoring here, the FBG interrogator was operated for 2 min once every hour. In each 2-min operation, the peak wavelengths at all FBGs are acquired with the sampling interval of $0.0014 \mathrm{~s}$ and saved in the control PC. In the processing, the 2-minlong peak wavelength data, which include around 84000 points, are averaged, and the temperature correlation is applied to the wavelengths of strain-FBG and temp-FBG in each sensing unit. The wavelength shift $\Delta \lambda_{B}$ at each data acquisition from the initial wavelength, which is the value in the initial data acquisition at 0:00 am on December 19, 2011, is calculated for the strain data.

Two of the eight strain data acquired in the period from December 19, 2011 to July 8, 2014 are shown in Figs. 3(a) and 3(b). The data now become time-series strain data with the time interval of one hour. It can be observed that the plots show clear seasonal and daily trends due mainly to the thermal expansion behavior of the bridge. ${ }^{(8)}$ It can be seen that the amplitudes of seasonal trends are both near $300 \mu \varepsilon$. This is understandable because the target bridge is located in the area where the air temperature becomes around $30^{\circ} \mathrm{C}$ in summer and around $0{ }^{\circ} \mathrm{C}$ in winter, and the thermal 


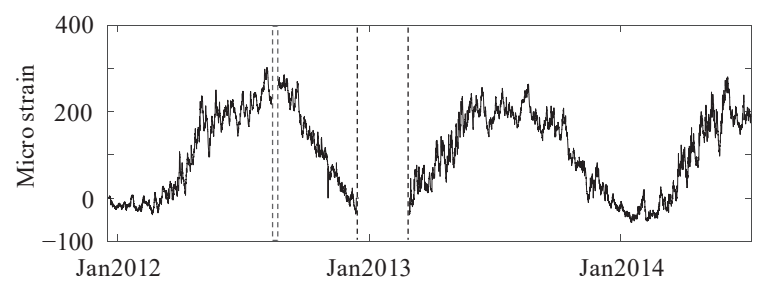

(a)

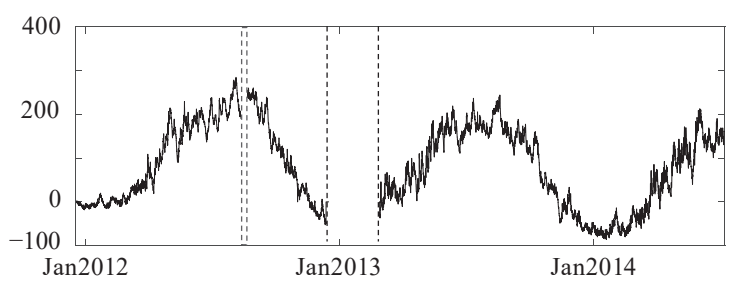

(b)

Fig. 3. Strain data from Dec 19, 2011 to July 8, 2014 at (a) L1-4 and (b) L2-1.

expansion coefficient of concrete is approximately $10 \times 10^{-6}{ }^{\circ} \mathrm{C}^{-1}$. During the long-term data acquisition, several periods of data missing due to unexpected trouble or operation mistakes could not be prevented. The longest period of data missing was about four months, from December 2012 to March 2013.

\subsection{Contributors to data quality}

In general, the performance of the fiber-optic structural monitoring system is affected by (i) the sensing elements, (ii) cables, and (iii) the light source, detector, and processing devices. ${ }^{(11)}$ In this study, those factors can be treated as (i) the FBGs, (ii) the cables for networking the sensing units, and (iii) the FBG interrogator and the data acquisition system.

The errors in measurement data are generally categorized as random error and bias error. With regard to (i) the strain-FBG and the temp-FBG in each sensing unit, the variabilities of the fiber properties in Eqs. (1) and (2) introduce random error. The FBG interrogator device (iii) also introduces random error because of the variabilities of electrical and optical components. This kind of random error cannot be eliminated in the measurement data. In addition, the performance of the FBG interrogator is also affected by the environmental conditions of the site, where the device is operated, such as temperature, humidity, dust, and so forth. These effects may cause data accuracy reductions, i.e., increases in random error and kinds of bias errors in data, or system failure in some cases. However, these errors can be prevented or improved by choosing an FBG interrogator device with the appropriate specifications for the monitoring site. In the case of this study, the monitoring site was inside the box girder; therefore, the devices were not directly exposed to the weather condition. However, because there was concern about the performance under low temperature in winter, the FBG interrogator and the control PC were installed inside a storage box. These devices were thus never down or out of order since the start of long-term monitoring.

The most significant factor that may affect the data quality, especially in the fiber-optic monitoring of civil structures, is (ii) cables, which include the cable network configuration and the attachment conditions of sensor devices on the structure. With regard to the former, the total length of the fiber network and the number of optical connections and their quality, such as the location and the number of fusion splice points, can affect the intensity and the spectrum profile of the reflection light. They are strongly related to the detection accuracy of the peak Bragg wavelengths of multipoint FBGs through a fiber. In this study, the network configuration was not changed since the installation of the monitoring system. Meanwhile, even though the optical fibers themselves are stable, the attachment condition of FBGs, i.e., the epoxy properties in this case, must be considered 
throughout the period of long-term monitoring. There must be initial variability in the epoxy properties due to uncertainties in the manufacturing process, e.g., mixture with hardener, amount of mixed resins in the attachment, and curing. If local stress occurs inside the epoxy as a result of those variabilities, it may cause the microbending of the fiber, promoting optical transmission

loss. Furthermore, the deterioration of epoxy, such as creep or cracks, which may be caused by, for instance, ultraviolet light, temperature variations, and humidity, cannot be ignored. The FBGs in this monitoring were attached inside the box girder and covered by the aluminum box; therefore, the effect of ultraviolet light is not considered to be significant. However, the possibility of deterioration due to other factors cannot be ignored as they might cause bias errors in the long-term data.

\section{Quality Evaluation of Long-Term Data}

On the basis of the considerations in the previous section, the qualities of acquired long-term data are evaluated from the viewpoints of reliability and stability. The stability includes the time variability of accuracy and sensitivity. For the verification here, only the two-minute data acquired at 2:00 am every day was used in order to deal with data that are little affected by traffic loads, and to remove daily temperature effect. The period of data to be analyzed was from May 26, 2012 to July 9, 2014, a 773-d period.

\subsection{Reliability}

The reliability is defined as the probability that the product will meet a set of specified properties for a given period of time in service. ${ }^{(1)}$ From this viewpoint, the data missing ratio in the 25 months becomes one of the measures indicating the reliability of the installed FBG monitoring system. The means of peak wavelengths in all FBGs are plotted in Fig. 4. Here, the strain-FBG and temp-FBG are indicated by "s" and " $\mathrm{t}$ ", respectively, and the range of the $y$-axis in all figures is set to be the same: $0.9 \mathrm{~nm}$. It can thus be seen that the amplitudes of periodic behaviors in the strain-FBGs are larger than those in the temp-FBGs. This indicates that the wavelength shifts in the strain-FBGs include both the mechanical strain of the thermal expansion deformation and the temperature effect on the fiber, i.e., the first and second terms in Eq. (2).

In all FBGs, there are two data-missing periods: from August 14 to 17 in 2012, and from December 13, 2012 to February 27, 2013, which is 81 days in total. The second data-missing period was actually due to the stoppage of the measurement system caused by a wrong setting of the data acquisition program. The other data-missing periods are from July 8 to September 28 in 2013 at only L2-3s, and from September 29 in 2013 at L2-4t. The total numbers of days with successful data acquisition are thus $609 \mathrm{~d}$ in L2-3t and $410 \mathrm{~d}$ in L2-4t.

The reliability of the strain monitoring system was then evaluated using the ratio of the number of days with successful data acquisition to the total number of days $(=773)$ at each sensing unit. The reliabilities were all $89.5 \%$ for the four sensor units on the L1 line (L1-1/2/3/4) and L2$1 / 2,78.8 \%$ for L2-3, and $53.0 \%$ for L2-4. It can be said that the reliabilities of the sensing units on network L1 are high because the data acquisitions were always successfully conducted all the time when the data acquisition system was appropriately operated. On the other hand, the reliabilities of the sensing units on network L2 decreased continuously with increasing distance from the FBG interrogator. From this result, one possibility that can be considered is that the peak 


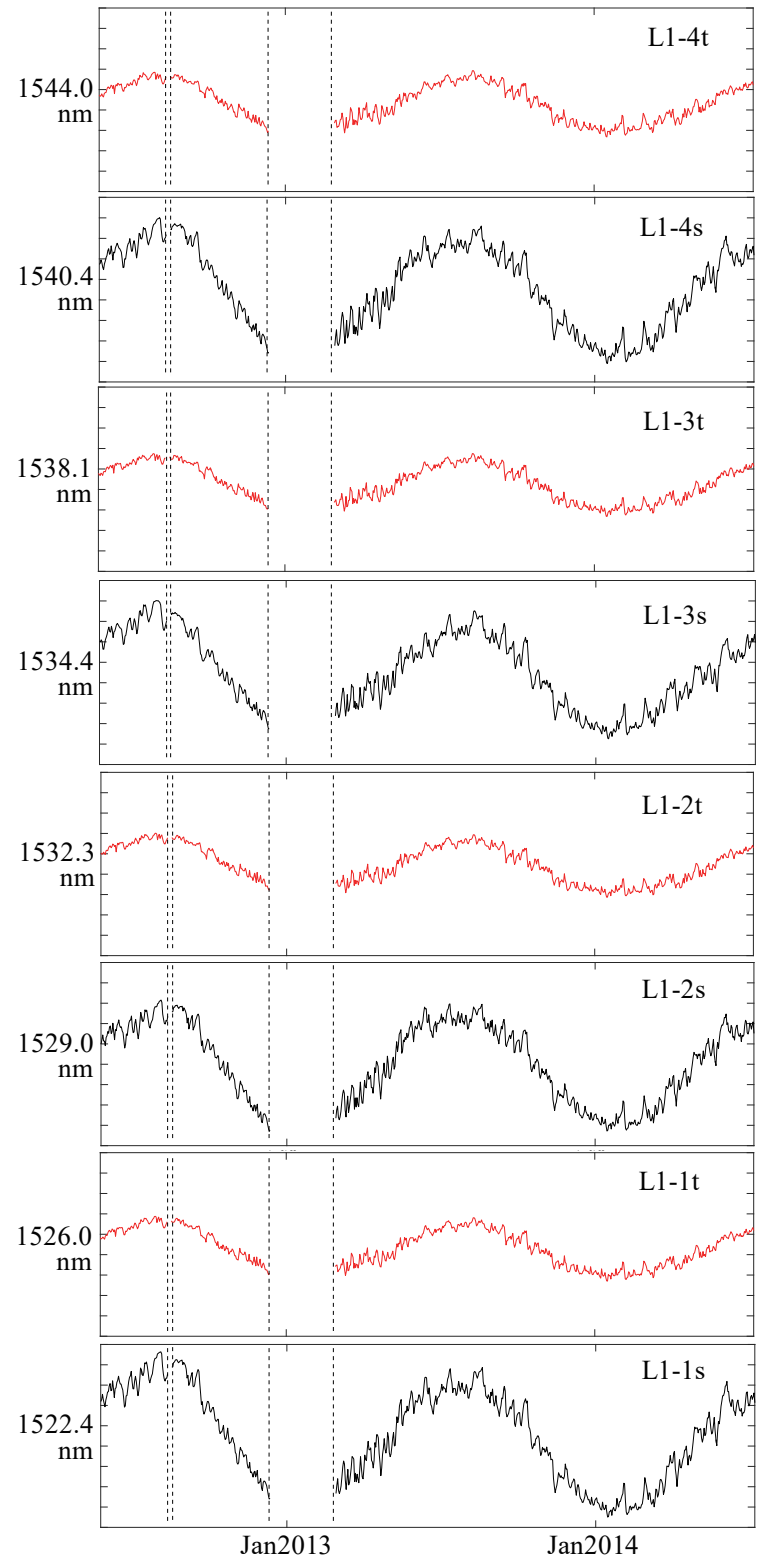

(a)

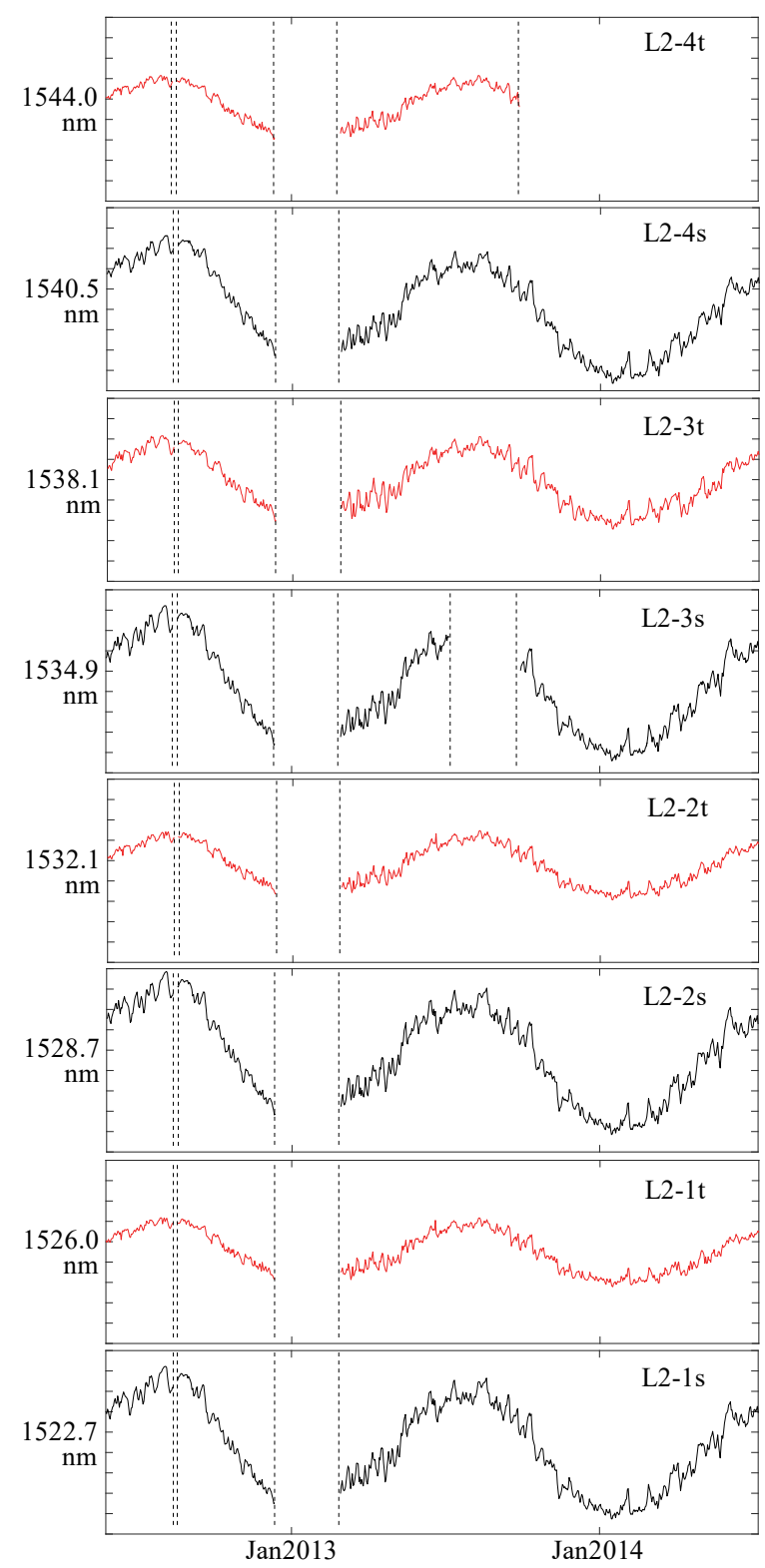

(b)

Fig. 4. (Color online) Mean of peak wavelength at each fiber line (a) L1 and (b) L2.

detection algorithm in the fiber line L2 did not work well because the reflection light spectrum was deteriorated. This actually could not be clarified if the intensity spectra of FBG reflection light had not been saved in the long-term monitoring. However, the amount of data of the intensity spectra through the range of input light wavelength is typically very large; therefore, they cannot be processed throughout the period of long-term monitoring. The result here brought to light the issues in the long-term monitoring, i.e., data storage, mining, and management. 


\subsection{Stability}

\subsubsection{Data accuracy}

Stability is the invariability of the required properties of data. In this viewpoint, the time variation of data accuracy, i.e., the standard deviation of the peak wavelength within each of the two-minute data acquisitions, was first verified. The long-term variations in standard deviations $\sigma$ in the period from 2012 to 2014 in all FBGs are shown in Fig. 5. Notice that, in all figures, the accuracies could be discussed in the range of $\sigma<0.003 \mathrm{~nm}$, which is $<2.5 \mu \varepsilon$ in strain and $<0.3{ }^{\circ} \mathrm{C}$ in temperature. Therefore, the data accuracies to be discussed here are comparable to those of data acquired in other widely used sensors, e.g., strain gage.

The most remarkable point in Fig. 5 is that a characteristic disturbance in the time variations of standard deviations is observed only in specific FBGs, L1-1s/t, L1-2s and L2-1s/t, L2-2s. The patterns of emerging large standard deviations were completely consistent within those FBGs, and there was no seasonal or daily trend. Furthermore, the standard deviations in the disturbance parts decrease with increasing distance from the FBG interrogator in each of the fiber lines L1 and L2. Figure 6 shows the time histories of peak wavelengths acquired in the two-minute data acquisition at L2-1s. Figure 6(a) shows the data acquired on June 28, 2014 with the standard deviation $\sigma$ $=0.0010 \mathrm{~nm}$, and Fig. $6(\mathrm{~b})$ shows the data that reveals the characteristic disturbance with the standard deviation $\sigma=0.0025 \mathrm{~nm}$. Both figures show random behaviors without any significant nonstationarity or bias behavior. From these points, it was considered that the characteristic disturbance of standard deviation occurred as a result of the variation in the performance of the FBG interrogator and data acquisition system including the light source behavior and the peak detection algorithm related to the profile of reflection light.

On the other hand, the nominal standard deviation levels, except to those in the disturbance parts, are consistent at slightly lower than $0.001 \mathrm{~nm}$ in all FBGs but L2-4t. Most standard deviations in L2-4t are distributed in the range of $0.0015-0.002 \mathrm{~nm}$. Notice that, in this L2-4t, the reliability was also lower than those of other FBGs, as mentioned in the previous section. This cannot be explained by the causes related to the FBG interrogator or the data acquisition system, which can affect the data quality globally. It was considered that the low data quality in L2-4t was due to more local issues such as the variability of FBG properties, the quality of FBG attachment using epoxy, or the light intensity loss at the points of fusion splicing in the cable networking.

Notice that, in some estimation procedures where the acquired data are used to provide information about the structural conditions, the standard deviation of data directly affects the accuracies of estimated values. The time variation of the standard deviation here can also be used to discuss the accuracy, i.e., the reliability, of information about structural conditions, which is also important information to be provided.

\subsubsection{Sensitivity}

The sensitivity of the strain sensing unit was evaluated using the correlation plots between the strain-FBG wavelengths and the temp-FBG wavelengths. The wavelengths of these two FBGs show strong positive correlations at every sensor unit, as shown in Fig. 7, which shows the plots for units L1-4 and L2-1. From these figures, no sensitivity changes were observed throughout the period from 2012 to 2014. 


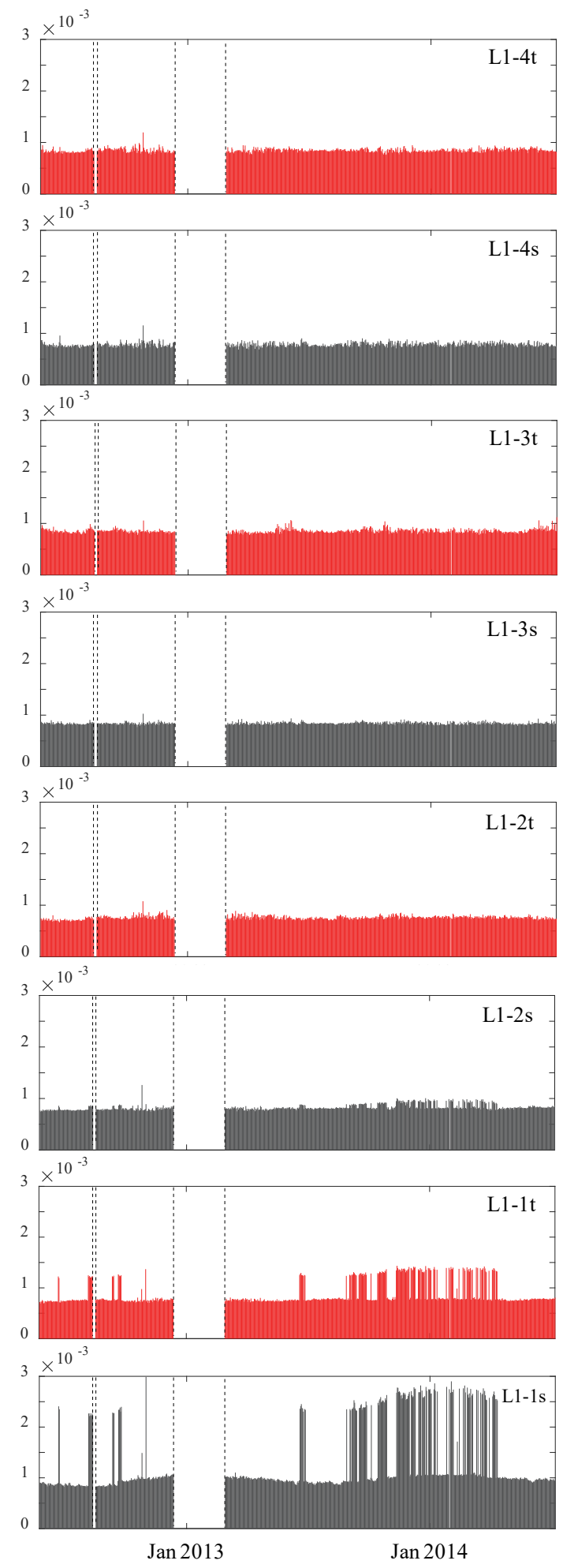

(a)

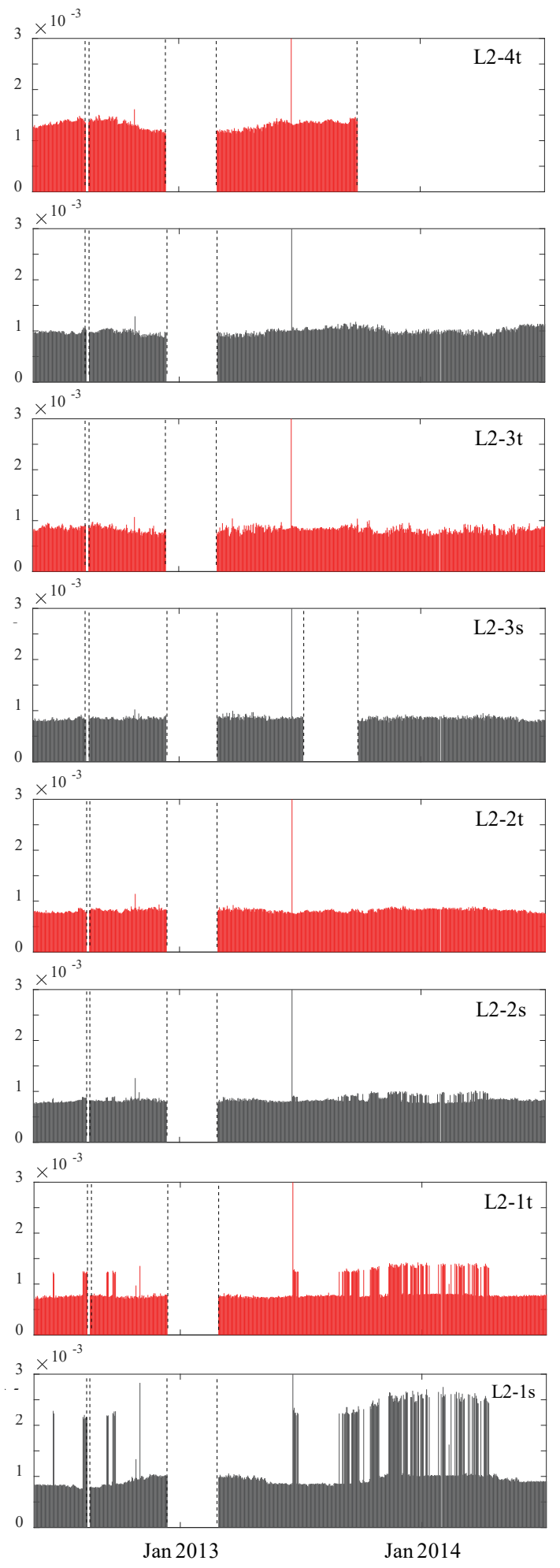

(b)

Fig. 5. (Color online) Standard deviation of peak wavelength (nm) at each fiber line: (a) L1 and (b) L2. 


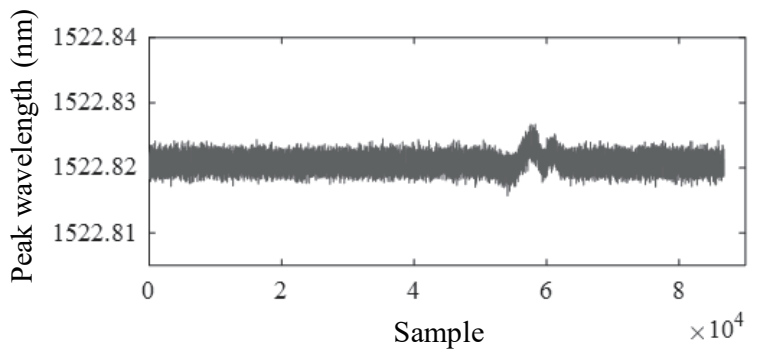

(a)

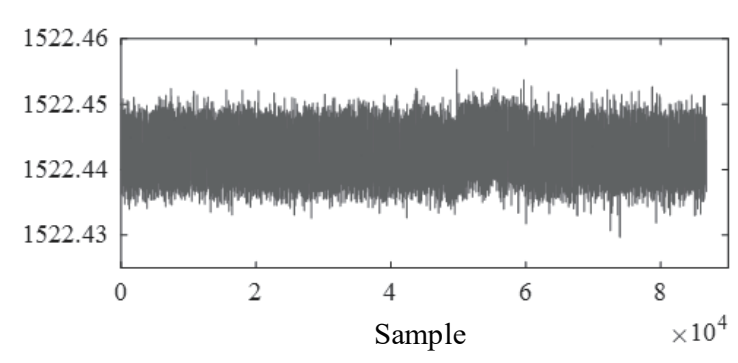

(b)

Fig. 6. Sampled peak wavelengths in the two-minute data acquisition at L2-1s at (a) 2:00 am on June 28, 2014 and (b) 2:00 am on April 8, 2014.

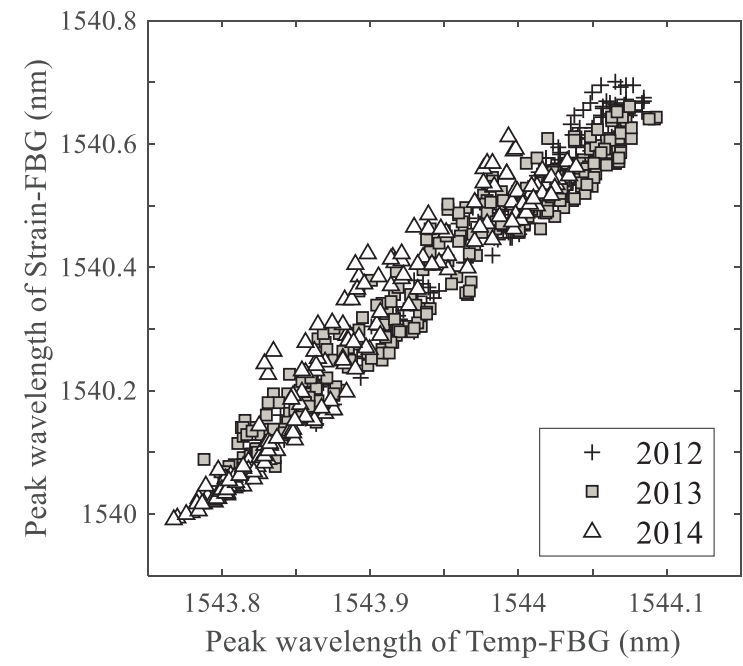

(a)

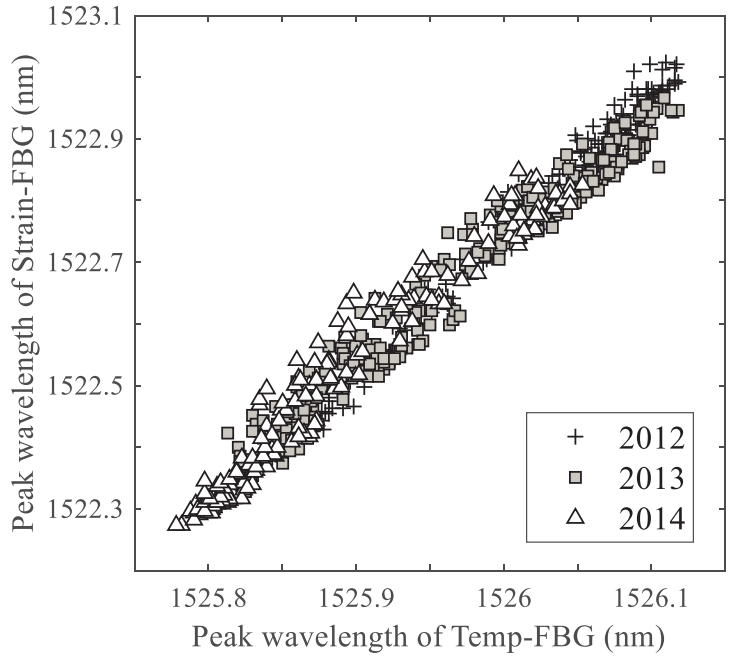

(b)

Fig. 7. Data sensitivity plot for (a) L1-4 and (b) L2-1.

For detailed evaluations, the slopes of linear regression functions and the correlation coefficients for each year were compared, as shown in Figs. 8(a) and 8(b), respectively. The slopes are basically determined by the coefficients $K_{\varepsilon}$ and $K_{T}$ in Eq. (2), and if the fabrications of both strain-FBG and temp-FBG are appropriate, the slopes may become constant. In Fig. 8(a), the slopes are thus distributed in almost the same order; however, the values in units L2-3 and L2-4 are slightly smaller than the others. One of the possible reasons was that the attachment condition of temp-FBGs was not appropriate. We see, in the mean-value plot of L2-3t in Fig. 4, that the amplitude of periodic behavior is slightly higher than those of other temp-FBGs. Therefore, there was a possibility that the temp-FBG at L2-3 was not appropriately attached, making it independent of mechanical strain. This then may have caused the different sensitivity compared with other sensing units.

Furthermore, in most sensor units, there is a tendency that the slopes in 2013 become lower than those in 2012, and they increase again in 2014. The reason for this tendency must be investigated through a more detailed analysis, taking into consideration both possibilities: the effects of structural behaviors and the sensing system performance itself. Meanwhile, the slopes in sensing unit L1-3 do not show this characteristic tendency throughout 2012-2014. Once the slope decreased in 2013, 


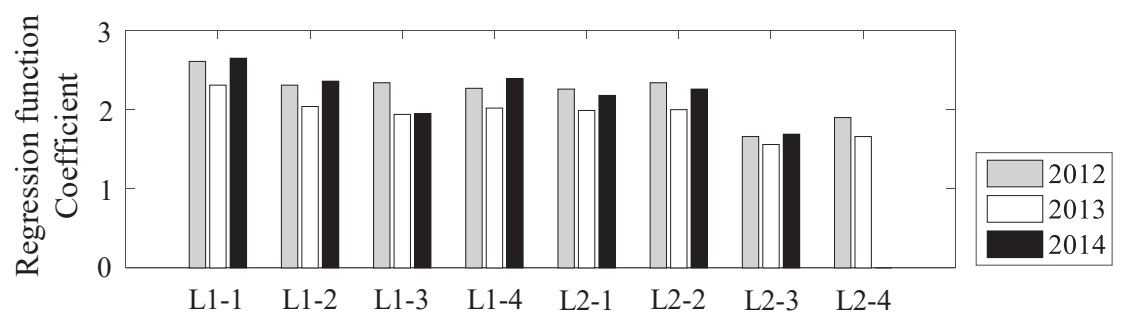

(a)

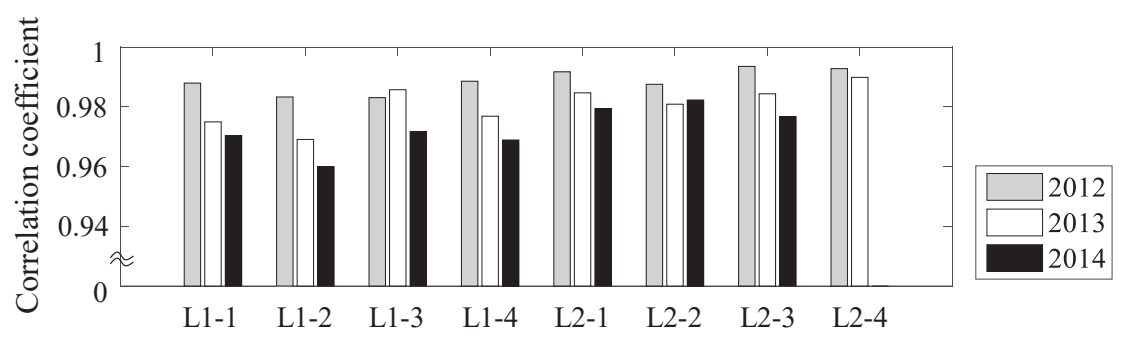

(b)

Fig. 8. Time variation of sensitivity determined from (a) slope of linear regression function and (b) linearity.

it did not recover to the same level as 2012 in 2014. One of possible reasons is that the sensitivity of the sensing unit decreased in L1-3 as a result of epoxy deterioration or other factors related to the attachment of FBGs on the structural surface.

Finally, the time variation of correlation coefficients in Fig. 8(b), i.e., the linearity of the sensing unit, gradually decreased in all sensing units. However, all the correlation coefficients themselves are very high $(>0.95)$ throughout the period of 2012-2014. It could then be concluded that no significant sensitivity reduction has yet occurred in this long-term monitoring.

\section{Conclusions}

The quality of data acquired in long-term bridge monitoring using FBG sensors was evaluated from the viewpoints of reliability and stability. After considering the error sources on the basis of the principle of sensors and the installation of the monitoring system, the reliability was discussed by examining the availability ratio of required data. It can be said that the reliability of the long-term data acquisition system including the FBG interrogator was very high; however, the reliability reduction at some FBGs could not be explained because it required the evaluation of the reflection light spectra. However, the difficulty of storing and handling large amounts of data in long-term monitoring was also pointed out. From the time variations of data accuracies at all FBGs, which was one way of evaluating stability, a characteristic disturbance in the standard deviations of random noise was recognized. It was then considered to be due to factors in the data acquisition system. Moreover, the attachment condition issues in some FBGs were also recognized as a result of the sensitivity analysis. The results and discussions in this paper will contribute to the appropriate use of long-term monitoring data in the condition assessment of existing civil infrastructures. The importance of analyzing the data quality in long-term SHM was then shown in this study. 


\section{Acknowledgements}

This study was supported by a research grant from the KAJIMA Foundation. In the fiber-optic bridge monitoring project, the author would like to thank Professor Hideaki Murayama from the University of Tokyo, for the establishment of the sensing system and cooperation in the long-term data acquisition.

\section{References}

1 K. Sugisaki, M. Abe, and S. Koshimizu: J. Jpn. Soc. Civil Eng., A1 69 (2013) 315 (in Japanese).

2 R. Zhang and E. Aktan: Sensing Issues in Civil Structural Health Monitoring (Springer, Netherlands, 2005) pp. 281-290.

3 E. J. Cross, J. M. W. Brownjohn, and K. Worden: Mech. Syst. Signal Process. 35 (2013) 16.

4 S. Soyoz and M. Q. Feng: Comput. Aided Civil Infrastruct. Eng. 24 (2009) 82.

5 H. C. Gomez, P. J. Fanning, M. Q. Feng, and S. Lee: Eng. Struct. 33 (2011) 2861.

6 A. Cury, C. Cremona, and J. Dumoulin: Mech. Syst. Signal Process. 33 (2012) 13.

7 P. Anderegg, R. Brönnimann, and U. Meier: J. Civil Struct. Health Monitoring 4 (2014) 69.

8 M. Nishio, J. Xu, and J. Mizutani: Proc. 10th Int. Workshop on Structural Health Monitoring 2 (2015) pp. 807-814.

9 B. Glisic and D. Inaudi: Fibre Optic Methods for Structural Health Monitoring (John Wiley \& Sons, England, 2008).

10 T. Saitoh, K. Nakamura, Y. Takahashi, H. Iida, Y. Iki, and K. Miyagi: IEEE Photonics Technol. Lett. 19 (17/20) (2007) 1616.

11 W. R. Habel: Sensing Issues in Civil Structural Health Monitoring (Springer, Netherlands, 2005) pp. 341-351.

\section{About the Author}

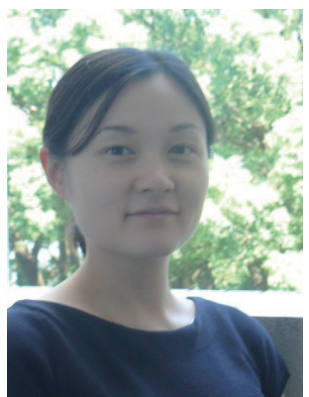

Mayuko Nishio received her B.S., M.S., and Ph.D. degrees from the University of Tokyo, Japan. From 2009 to 2010, she was a visiting scholar as a JSPS fellow at the Los Alamos National Laboratory, USA, and then a research fellow at the University of Tokyo from 2010 to 2011. Since 2011, she has been an associate professor at Yokohama National University. One of her research interests is the structural health monitoring of civil infrastructures using fiber-optic sensors. 